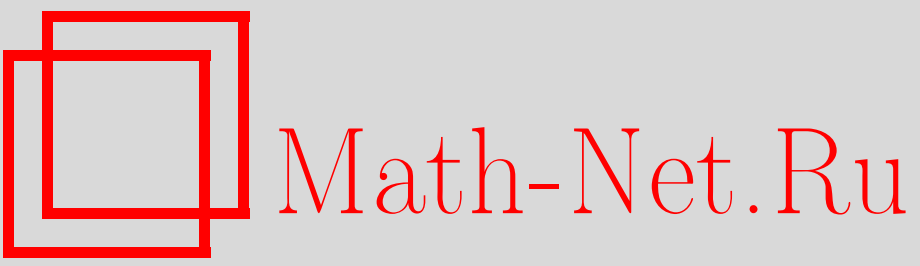

С. М. Натанзон, Теорема типа Харнака для семейства комплексно изоморфных вещественных алгебраических кривых, УМН, 1997, том 52, выпуск 6, 173-174

DOI: https://doi.org/10.4213/rm908

Использование Общероссийского математического портала Math-Net.Ru подразумевает, что вы прочитали и согласны с пользовательским соглашением

http://www.mathnet.ru/rus/agreement

Параметры загрузки:

IP: 3.89.197.203

26 апреля 2023 г., 13:11:29 


\title{
ТЕОРЕМА ТИПА ХАРНАКА ДЛЯ СЕМЕЙСТВА КОМПЛЕКСНО ИЗОМОРФНЫХ ВЕЩЕСТВЕННЫХ АЛГЕБРАИЧЕСКИХ КРИВЫХ
}

\author{
C. М. НАТАНЗОН
}

Пусть $X$ неособая неприводимая вещественная алгебраическая кривая рода $g$. Она назьвается ориентируемой (или типа I в терминологии Клейна), если множество ее вещественных точек $\mathbb{R}(X)$ делит множество ее комплексных точек $\mathbb{C}(X)$. Рассмотрим теперь семейство $X_{1}, \ldots, X_{n}$ попарно неизоморфных ориентируемых вещественных алгебраических кривых рода $g$, комплексификации которых $\mathbb{C}\left(X_{i}\right)$ изоморфны как комплексные алгебраические кривые. Все такие семейства легко описать для $g \leqslant 1$ и далее мы считаем, что $g>1$. Согласно [1] $(n-4) 2^{n-3} \leqslant g-1$ и эта оценка точна.

Согласно теореме Харнака вещественные точки $\mathbb{R}\left(X_{i}\right)$ распадаются на простые замкнутые контуры, называемые овалами. Их число $\left|X_{i}\right|$ не превосходит $g+1$ и эта оценка точна. Наша цель - оценить число $\sum_{i=1}^{n}\left|X_{i}\right|$. Очевидная оценка $\left|X_{1}\right|+\left|X_{2}\right| \leqslant 2 g+2$ точна и достигается на вещественных гиперэллиптических кривых. Согласно [2] $\sum_{i=1}^{3}\left|X_{i}\right| \leqslant 2 g+4$ и $\sum_{i=1}^{4}\left|X_{i}\right| \leqslant$ $2 g+8$. В этой статье мы дадим набросок доказательства.

TeOpema.

$$
\sum_{i=1}^{n}\left|X_{i}\right| \leqslant 2 g-(n-9) 2^{n-3}-2 \leqslant 2 g+30,
$$

причем первая оченка точна при каждом $n$ для бесконечного числа $g$.

Доказательство основано на работе [3], согласно которой семейство $X_{1}, \ldots, X_{n}$ порождает конечную группу Кокстера и классификации [4] конечных групп Кокстера.

Напомним, что вещественная алгебраическая кривая порождает пару $(\mathbb{C}(X), \tau)$, где $\tau: \mathbb{C}(X) \rightarrow \mathbb{C}(X)$ - инволюция комплексного сопряжения, причем множество

$$
\mathbb{R}(X)=\{p \in \mathbb{C}(X) \mid \tau p=p\} .
$$

Группа Кокстера возникает следующим образом. Рассмотрим комплексную алгебраическую кривую $P$, которая комплексно изоморфна кривьп $\mathbb{C}\left(X_{i}\right)$. Комплексные многообразия $P$ и $\mathbb{C}\left(X_{i}\right)$ являются римановьми поверхностями рода $g=g(P)$. Изоморфизмы между $\mathbb{C}\left(X_{i}\right)$ и $P$ индуцируют биголоморфные отображения $\varphi_{i}: \mathbb{C}\left(X_{i}\right) \rightarrow P$. Они переводят инволюции комплексного сопряжения $\widetilde{\tau}_{i}: \mathbb{C}\left(X_{i}\right) \rightarrow \mathbb{C}\left(X_{i}\right)$ в антиголоморфные инволюции $\tau_{i}: P \rightarrow P$. Попарная неизоморфность $X_{i}$ эквивалентна попарной несопряженности инволюций $\tau_{i}$ в группе $\operatorname{Aut}(P)$ голоморфных и антиголоморфных автоморфизмов $P$. Множество $P^{\tau_{i}}=\left\{p \in P \mid \tau_{i} p=p\right\}$ распадается на $\left|\tau_{i}\right|=\left|X_{i}\right|$ простых контуров. Таким образом, неравенство (1) сводится к неравенству

$$
\sum_{i=1}^{n}\left|\tau_{i}\right| \leqslant 2 g-(n-9) 2^{n-3}-2
$$

для произвольного набора попарно несопряженных в $\operatorname{Aut}(P)$ антиголоморфных инволюций $\tau_{i}: P \rightarrow P$ таких, что $P^{\tau_{i}}$ делит $P$.

Лемма 1. Существуют антиголоморфные инволюции $\alpha_{i}: P \rightarrow P(i=1, \ldots, n)$ такие, что $P^{\alpha_{i}}$ делят $P, \alpha_{i} \neq \alpha_{j}$ при $i \neq j, \alpha_{i} \alpha_{j}=\alpha_{j} \alpha_{i} u \sum_{i=1}^{n}\left|\tau_{i}\right| \leqslant \sum_{i=1}^{n}\left|\alpha_{i}\right|$.

СХеМА ДОКАЗАТЕЛЬСТВА. Согласно [3] инволюции $\tau_{1}, \ldots, \tau_{n}$ порождают группу Кокстера $\widetilde{W}$, используя этот факт и теорию групп Кокстера [4], можно доказать существование множества инволюций $B=\left(\beta_{1}, \ldots, \beta_{n}\right)$, порождающих систему Кокстера $(W, B)$, и таких, что $\beta_{i}$ сопряжены $\tau_{i}$ в $\widetilde{W}$. Положим $\alpha_{i}=\beta_{i}$, если $\beta_{i}$ принадлежит центру группы $W$. Элементы $\beta_{i} \beta_{j}$ имеют четную степень, поскольку $\beta_{i}$ и $\beta_{j}$ не сопряжены в $W$. Из этого факта и классификации систем Кокстера [4] следует, что непринадлежащие центру группы $W$ инволюции $\beta_{i}$ разбиваются на пары $\left(\beta_{i}, \beta_{j}\right)$, где $\beta_{j}$ коммутирует с $B \backslash \beta_{i}$. Пусть $\gamma=\left(\beta_{i} \beta_{j}\right)^{m}$, где $2 m$ - порядок $\left(\beta_{i} \beta_{j}\right)$. Для $\left|\beta_{i}\right| \geqslant\left|\beta_{j}\right|$ положим $\alpha_{i}=\beta_{i}, \quad \alpha_{j}=\gamma \beta_{i}$. Тогда $\sum_{i=1}^{n}\left|\alpha_{i}\right| \geqslant \sum_{i=1}^{n}\left|\tau_{i}\right|$. 
Таким образом, нам осталось доказать неравенство

$$
\sum_{i=1}^{n}\left|\alpha_{i}\right| \leqslant 2 g-(n-9) 2^{n-3}-2
$$

для множества $A=\left(\alpha_{1}, \ldots, \alpha_{n}\right)$ коммутирующих антиголоморфиных инволюций римановой поверхности $P$ таких, что $P^{\alpha_{i}}$ делит $P$.

Это делается индукцией по $g$ с помощью двух лемм.

Лемма 2. Если неравенство дожазано для поверхностей рода $<$ я и одна из связньи компонент множества $\bigcup_{\alpha_{i} \in A} P^{\alpha_{i}}$ содержится в $P^{\alpha_{n-1}} \cup P^{\alpha_{n}}$, то неравенство верно для поверхностей рода $g$.

Лемма 3. Если среди компонент связности множества $\bigcup_{\alpha_{i} \in A} P^{\alpha_{i}}$ нет компонент, содержащихся в множествах вида $P^{\alpha_{i}} \cup P^{\alpha_{j}}(i, j=1, \ldots, n)$, то неравенство (3) выполняется.

Лемма 2 доказьвается с помощью "хирургии" поверхностей, то есть разрезами и склейками вдоль $P^{\alpha_{i}}$. Дадим набросок доказательства леммы 3 . Пусть $W$ - группа, порожденная $A$. Граница связной компоненты $K \in P \backslash \bigcup_{\alpha_{i} \in A} P^{\alpha_{i}}$ распадается на $k$ связных компонент. Каждая из них состоит из отрезков $\ell_{i 1}, \ldots, \ell_{i m_{i}}$, где $\ell_{i j} \in P^{\sigma(i, j)}$. Пусть $t_{i}$ число разных элементов группы $W$ среди $\sigma(i, 1), \ldots, \sigma\left(i, m_{i}\right)$. По условию $t_{i} \geqslant 3$ и $\sum_{i=1}^{n} t_{i} \geqslant n$. Для $n \in \mathbb{Z}$ положим $\sigma\left(i, j+n m_{i}\right)=\sigma(i, j)$. Рассмотрим

$$
L_{i}^{1}=\left\{\ell_{i j} \mid \sigma(i, j-1)=\sigma(i, j+1)\right\}, \quad L_{i}^{2}=\left\{\ell_{i j} \mid \sigma(i, j-1) \neq \sigma(i, j+1)\right\} .
$$

Обозначим через $s_{i}$ число элементов в множестве $L_{i}^{2}$. Тогда $s_{i} \geqslant t_{i}-1$.

Рассмотрим естественную проекцию $\varphi: P \rightarrow P / W=\widetilde{P}$. Прообраз $\varphi^{-1}\left(\ell_{i j}\right)$ состоит из овалов инволюции $\sigma(i, j)$, число которых равно $2^{n-2}$, если $\ell_{i j} \in L_{i}^{1}$ и $2^{n-3}$, если $\ell_{i j} \in L_{i}^{2}$. Таким образом,

$$
\sum_{i=1}^{n}\left|\alpha_{i}\right|=\sum_{i=1}^{n} s_{i} \cdot 2^{n-3}+\left(m_{i}-s_{i}\right) 2^{n-2} \leqslant \sum_{i=1}^{k} m_{i} \cdot 2^{n-2}+(k-n) \cdot 2^{n-3} .
$$

Кроме того, согласно теореме Римана-Гурвица $4 g-4=2^{n}\left(2 \widetilde{g}-4+2 k+\frac{1}{2} \sum_{i=1}^{k} m_{i}\right)$, где $\widetilde{g}-$ род $\widetilde{P}$. Сопоставление этих неравенств дает $\sum_{i=1}^{n}\left|\alpha_{i}\right|-2 g \leqslant-(n-9) 2^{n-3}-2$.

Точность неравенства (2) доказывается построением поверхности $P$ с помощью неевклидовых кристаллограффических групп. А именно, пусть $\Lambda=\{z \in \mathbb{C} \mid$ In $z>0\}$ - верхняя полуплоскость, наделенная стандартной структурой геометрии Лобачевского. Рассмотрим прямоугольник геометрии Лобачевского с несоизмеримыми сторонами $\ell_{1}, \ldots, \ell_{n+2 m} \in \Lambda$. Пусть $\sigma_{i}$ - отражение в стороне $\ell_{i}$ в смысле геометрии Лобачевского. Пусть $G$ - группа, порожденная $\sigma_{i}$. Пусть $\psi: G \rightarrow\left(\mathbb{Z}_{2}\right)^{n}$ - эпиморфизм такой, что $\psi\left(\sigma_{1}\right), \ldots, \psi\left(\sigma_{n-1}\right), \psi\left(\sigma_{n+1}\right)$ - образующие $\left(\mathbb{Z}_{2}\right)^{n}$ и $\psi\left(\sigma_{n+2 j}\right)=\psi\left(\sigma_{2}\right), \psi\left(\sigma_{n+2 j+1}\right)=\psi\left(\sigma_{n+1}\right)(j=0, \ldots, m)$. Тогда $P=\Lambda / G-$ компактная риманова поверхность рода $g=2^{n-3}(n+2 m-4)+1$. Инволюции $\sigma_{i}$ порождают на $P$ антиголоморфине инволюции $\tau_{i}(i=1, \ldots, n-1, n+1)$. Ввиду несоизмеримости $\ell_{i}$ инволюции $\tau_{i}$ не сопряжены в группе $\operatorname{Aut}(P)$, и, следовательно, вещественные кривые $\left(P, \tau_{i}\right)$ попарно не изоморфны. С другой стороны, можно доказать, что $\left|\tau_{1}\right|+\cdots+\left|\tau_{n-1}\right|+\left|\tau_{n+1}\right|=(n-1)^{2 n-1}+(2 m+1) 2^{n-2}$, откуда $\left|\tau_{1}\right|+\cdots+\left|\tau_{n-1}\right|+\left|\tau_{n+1}\right|=2 g-(n-9) 2^{n-3}-2$.

\section{СПИСОК ЛИТЕРАТУРЫ}

[1] Bujalance E., Gromadzki G., Singerman D. // Proc. Amer. Math. Soc. 1994. V. 120. № 2. P. 507-513. [2] Натанзон C. M. // Труды МMO. 1988. T. 51. C. 3-53. [3] Натанзон С. М. // УМН. 1996. Т. 51. № 6. С. 215-216. [4] Бурбаки Н. Группы и алгебры Ли. М.: Мир, 1972. 